
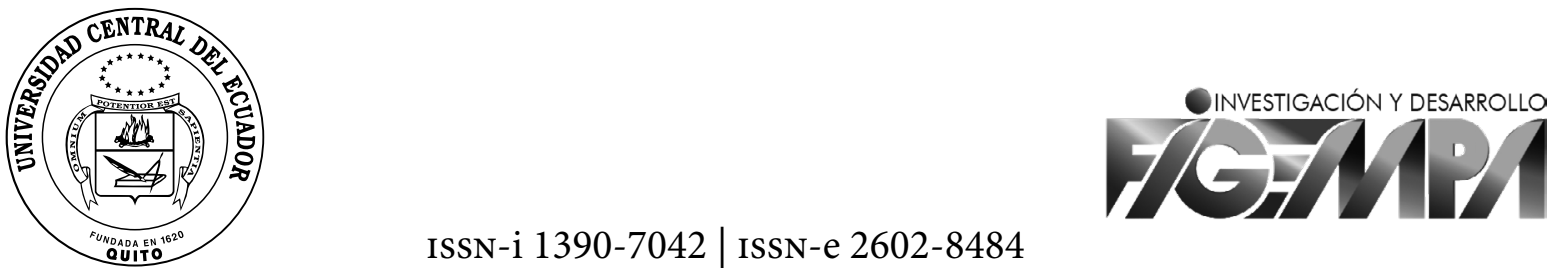

ISSN-i 1390-7042 | ISSN-e 2602-8484

\title{
ANÁLISIS DE COSTOS OPERATIVOS EN PEQUEÑA MINERÍA Y MINERÍA ARTESANAL EN NAMBIJA
}

\author{
Analysis of operating costs in small-scale mining \\ and artisanal mining in Nambija
}

\author{
Cynthia Rojas Villacís \\ Instituto de Investigación Geológico y Energético \\ cynthia.rojas@geoenergia.gob.ec
}

\author{
Cristian Zúñiga Arrobo \\ Instituto de Investigación Geológico y Energético \\ cristian.zuniga@geoenergia.gob.ec
}

Recibido: 10 de septiembre de 2020

Aprobado: 18 de diciembre de 2020 ARTÍCULO ORIGINAL

DOI: $10.29166 /$ REVFIG.V1I2.2568

Palabras Clave Minería artesanal, pequeña minería, Nambija, minería subterránea, costos operativos, análisis de costo, costo horario.

KEYWORDS Artisanal mining; small mining; Nambija; underground mining; operating costs; Cost analysis; hourly cost.

\section{RESUMEN}

En el desarrollo de la actividad minera en pequeña minería y minería artesanal en Ecuador, en varios casos no se lleva un registro de los costos operativos que demanda cada tarea realizada en el ciclo de trabajo minero, en consecuencia, no se conoce al final cuáles son los costos de inversión en contraste con la recuperación económica alcanzada. El objetivo de este trabajo fue analizar los costos operativos en cada tarea realizada en el ciclo de trabajo minero en tres labores del sector de Nambija, con la finalidad de evidenciar cuáles son los costos que se ocupa en realizar la tarea de perforación, voladura, ventilación, desate, limpieza del frente de trabajo, cargado y transporte del material arrancado que comprenden un ciclo minero de trabajo. En el análisis de costos se ha considerado el número de trabajadores mineros que realiza cada tarea y cuánto perciben en salario por jornada o por tarea realizada, el tiempo que se demoran en realizar dicha actividad, la maquinaria y los equipos que utilizan; la cantidad y su capacidad, el consumo de energía, combustibles, lubricantes por hora de operación, sus repuestos y reparaciones, los insumos como conexiones de agua, aire y energía utilizados y sus costos y las tareas auxiliares aplicadas previas o posteriores a la tarea a desarrollar. En la estimación de los costos horarios de los equipos se ha tomado en cuenta (a) costos indirectos o de propiedad: amortización y seguros, y (b) costos directos de funcionamiento: consumo de energía eléctrica, combustible, lubricantes, grasas y filtros, reparaciones, neumáticos o elementos de mayor desgaste, salario de operadores o trabajadores. Las tres labores mineras analizadas en el sector de Nambija corresponden a una mina artesanal que desarrolla trabajos en un solo frente, alquilan compresor a diésel para conectar un martillo perforador para ejecutar los trabajos, tienen equipos básicos y limitados - casi no cuentan con repuestos-, posee vagones de pequeña capacidad 0,75 a 1 tonelada, el personal de mina ejecuta varias actividades simultáneamente y el salario percibido es el más bajo del sector; la segunda labor minera analizada se caracteriza por tratar de ejecutar trabajos de pequeña minería en uno y máximo dos frentes de trabajo, ya que posee un compresor propio a diésel para conectar dos martillos de perforación simultáneamente, tienen personal dedicado a realizar tareas específicas, posee vagones de capacidad 1 tonelada; y la tercera labor minera realizan actividades de pequeña minería, trabajan en dos y tres frentes - cuando obtienen buen material-, poseen un compresor propio de funcionamiento eléctrico con capacidad para conectar 3 
martillos perforadores simultáneamente, poseen vagones de 2 y hasta 3 toneladas de capacidad y tienen personal para realizar actividades específicas en cada puesto de trabajo. Como resultado del análisis de los costos operativos se tiene el costo por cada tarea del ciclo de trabajo minero en dólares/hora (USD/h) y el porcentaje (\%) de cada actividad en función al costo total, información que sirve para comparar el comportamiento económico de tres tipos de labores mineras en minería artesanal y pequeña minería.

\begin{abstract}
In the development of the mining activity in small mining and artisanal mining in Ecuadorian in several cases a record of the services performed is not carried out of investment in contrast with the economic recovery achieved. The objective of this work has focused on the services in each task in the work cycle at work in the sector of Nambija, in order to highlight the work in which the task of drilling, blasting, ventilation, untie is performed, cleaning of the work front, loading and transport of the arranged material that worked in a mining work cycle. In the analysis of costs has been considered the number of mining workers who perform each task and how much they receive in salary per day, or per task performed, the time they take to carry out this activity, the machinery and equipment they use; the quantity and capacity, the consumption of energy, fuels, lubricants per hour of operation, their spare parts and repairs, the inputs such as water, air and energy connections used and their costs and auxiliary tasks applied before or after the task to develop. In estimating the hourly costs of the equipment (a) indirect or property costs: amortization and insurance, and (b) direct operating costs: consumption of electric power, fuel, lubricants, greases and filters, repairs, tires or elements of greater wear, salary of operators or workers. The three mining works analyzed in the sector of Nambija correspond: to an artisanal mine that develops works on a single front, they rent a diesel compressor to connect a hammer driller to execute the works, they have basic and limited equipment - almost do not have spare parts- , has small capacity wagons of 0.75 to 1 ton, the mine staff performs several activities simultaneously and the perceived salary is the lowest in the sector, the second mining work analyzed is characterized by trying to execute small mining works in one and maximum two working fronts already has a diesel own compressor to connect two drilling hammers simultaneously, they have personnel dedicated to perform specific tasks, has wagons of capacity 1 ton, and the third mining work perform activities of small mining, work in two and three fronts - when they get good material-, they have their own electric compressor c On ability to connect 3 drilling hammers simultaneously, have wagons of 2 and up to 3 tons capacity and have personnel to perform specific activities in each job. As a result of the analysis of the operating costs, we have the cost for each task of the mining work cycle in dollars / hour (USD/h) and the percentage (\%) of each activity based on the total cost, information that is used to compare the economic behavior of three types of mining work in artisanal mining and small mining.
\end{abstract}

\section{INTRODUCCIÓN}

En la provincia de Zamora Chinchipe, parroquia San Carlos de las Minas, en el sector de Nambija, se desarrolla actividad minera en la existen varias labores que extraen material rocoso ${ }^{1}$ que presenta contenido mineral ${ }^{2}$ de interés económico; entre los principales, el oro y la plata. Estas labores mineras tienen dife- rentes dimensiones de trabajo, avance, profundidad, grado de tecnificación, entre otros, que no han sido desarrollados siguiendo algún orden o planificación.

Las labores mineras presentan diferentes grados de inversión y diferentes tipos de asociaciones o dueños con complejo grado de participación económica y estructuralmente conectadas entre sí. 
En Ecuador, la minería artesanal y de sustento por su capacidad de producción se considera que para minerales metálicos se puede extraer hasta 10 toneladas por día en minería subterránea (Ley de Minería, Ecuador, 2018).

Para minería artesanal, la maquinaria y equipos para la obtención de minerales metálicos utilizados en explotación subterránea en minado son: compresor con una capacidad de $180 \mathrm{cfm}$ (pies cúbicos por minuto), martillo perforador neumático, martillo perforador eléctrico, barrenos de perforación de hasta 1,50 metros; para clasificación, carga y transporte: trituradora de mandíbulas, winche, carros de mina; en equipos auxiliares: bombas de agua, ventilador y extractor de gases y generador eléctrico (Agencia de Regulación y Control Minero, ARCOM, 2013).

La mayoría de las minas creadas en este sector se ha desarrollado artesanalmente y desde el 2 de octubre 2015 fue otorgado el régimen de pequeña minería (Ministerio de Minería, 2015).

En pequeña minería para minerales metálicos se puede extraer hasta 300 toneladas por día en minería subterránea (Ley de Minería, Ecuador, 2018).

\section{PLANTEAMIENTO DEL PROBLEMA}

La puesta en marcha de un proyecto minero compromete contar con la información técnica ${ }^{3}$ y económica ${ }^{4}$ que permita evaluar ${ }^{5}$ si la actividad desarrollada es rentable respecto a las inversiones destinadas al capital fijo ${ }^{6} \mathrm{y}$ circulante; ${ }^{7}$ el fijo que generan los fondos necesarios para adquisición de terrenos, estudios previos, infraestructura, diseño, entre otros; en tanto que el circulante es utilizado para realizar compra de insumos, pago de obreros, así como todo compromiso de desembolso para poner en marcha la actividad productiva. ${ }^{8}$

La actividad minera artesanal y de pequeña minería en Ecuador muchas veces no contempla o no lleva un registro adecuado de los costos operativos ${ }^{9}$ que demanda esta actividad y en su mayoría desconocen si al final del trabajo en la recuperación ${ }^{10}$ y comercialización ${ }^{11}$ de los metales extraídos de oro y plata, están obteniendo una ganancia respecto a los costos utilizados para obtener el producto final.

El alcance del presente estudio es determinar los costos operativos ${ }^{12}$ utilizados en las tareas del ciclo de trabajo minero en cada una de las labores analizadas; una vez obtenidos estos insumos, las labores mineras tendrán la capacidad de comparar y verificar la rentabilidad en cada uno de sus proyectos.

\section{OBJETIVO}

Realizar el análisis de los costos operativos en las tareas del ciclo de trabajo minero (perforación, vo- ladura, ventilación, saneamiento, limpieza, cargado y acarreo), en las tareas auxiliares (instalación de tubería de agua, aire y conexiones eléctricas), y, costos adicionales (energía eléctrica y equipos de protección personal), en el frente de producción en tres labores mineras del sector de Nambija.

\section{MATERIAL Y MÉTODOS}

Las actividades para obtener información:

- Revisión bibliográfica sobre las tareas del ciclo de trabajo en minas subterráneas e información sobre evaluación económica de proyectos, costos de operación y métodos de estimación;

- Definición de parámetros técnicos económicos a ser levantados mediante fichas de diagnósticos;

Elaboración de fichas técnicas de diagnóstico;

- Preparación de logística para salida de campo;

- Acercamiento y socialización con los operadores mineros;

- Recolección de información en la ficha técnica de diagnóstico;

- Validación de información y procesamiento de datos para el cálculo de costos; y

- Elaboración de cuadros estadísticos de los aspectos técnicos y económicos;

\section{CÁLCULO DE COSTO HORARIO}

El cálculo del costo horario corresponde al valor económico del costo de propiedad ${ }^{13}$ más los costos de operación dividido para la producción horaria.

El costo horario de propiedad está constituido por: amortización, interés del capital invertido, seguros e impuestos, lo que constituye los costos indirectos. - Amortización de equipos: disminución en el valor de la propiedad debido al uso, deterioro y a la caída en desuso de un bien.

$$
A=\frac{p a-v r-v n}{v u} \quad \text { EC. } \mathbf{1}
$$

Donde:

A: amortización de equipos;

pa: precio de adquisición;

vr: valor residual;

vn: valores neumáticos, $\mathrm{y}$

vu: vida útil.

- Amortización lineal: suma a amortiza dividido para las horas de vida estimada hasta la sustitución por venta o desecho del equipo.

- Precio de adquisición (pa) depende de:

- precio en el marcado nacional,

- repuestos, y 
- costos por transporte y montaje.

INVERSIÓN MEDIA (IM)

Se calcula con la siguiente fórmula.

$\operatorname{Im}=\frac{p a *(n+1)}{2 n} \quad$ EC. 2

Donde:

n: número de años de amortización.

- Valor residual o reventa ( $v r)$ : es la estimación del valor de mercado al final de la vida útil del equipo (20\% del costo de adquisición del equipo).

- Vida útil del equipo (vu): depende del estándar de fabricación y de las condiciones de trabajo.

- Cargas indirectas: inversión media anual dividida para el número de horas de trabajo al año del equipo. - Costos directos de funcionamiento ${ }^{14}$ o costos de operación ${ }^{15}$ depende del consumo de energía eléctrica, combustibles, lubricantes, reparaciones, neumáticos o elementos de mayor desgaste y el pago de operadores (trabajadores).

\section{COSTO DEL COMBUSTIBLE Y ENERGÍA}

$$
C c b=0.3^{\star} k W^{\star} F t^{\star} P c b \quad \text { EC. } 3
$$

Donde:

Ccb: costo horario por el uso de combustible (USD/h), $\mathrm{kW}$ : potencia nominal del equipo,

Ft: factor teórico por el uso de combustible,

Pcb: precio del combustible.

En equipos eléctricos, los consumos horarios se calcularon en función de la potencia instalada:

$$
\frac{k W h}{h}=k W * F c \quad \text { EC. } 4
$$

Donde:

$\mathrm{kW}=$ potencia nominal en $\mathrm{kW}$

$\mathrm{Fc}=$ factor de carga, normalmente 0.7

- Lubricantes, grasas y filtros: aceites de motor, sistema hidráulico, y grasa para los elementos de contacto metal-metal; se consideró como un porcentaje del costo horario de combustible, generalmente entre (10 y 20\%). - Reparaciones: costos sobre averías de equipos, considerando materiales y mano de obra.

$$
\frac{(\text { Pad }- \text { Prep })}{(h v)} * \text { Frep } \quad \text { EC. } 5
$$

Donde:
Pad: precio de adquisición,

Prep: precio de la parte a ser reparada, hv: horas de vida útil, Frep: factor de reparación.

El Factor de reparación fluctúa entre 0.7 a 1.1 dependiendo de las condiciones de trabajo.

- Neumáticos y elementos de recambio: depende del costo total y la vida útil, considerados el cálculo del costo horario de la maquinaria.

- neumáticos de los carros mineros (vagones)

- barrenos integrales (un solo cuerpo)

- filtros de aire de compresores

- Operadores; salarios que perciben los trabajadores en el sector, por jornada o pago diario.

El índice de utilización del personal: es la relación del número total del personal presente en la mina versus el número de personas que realizan específicamente la tarea designada; este índice fluctúa de 0 a 1 o 0 a $100 \%$, con este dato se realiza la corrección al costo final del ciclo de trabajo denominándolo como costo por tiempo muerto en mano de obra.

\section{RESULTADOS}

\section{LABOR MINERA A}

Coordenadas

\begin{tabular}{cccc}
\hline WGS 84 & E & N & h(m.s.n.m.) \\
\hline & 745629 & 9549953 & 1923 \\
\hline
\end{tabular}

Número total de personas que trabajan en la mina: 4 personas en 1 frente de trabajo, en 1 turno diario.

\section{LABOR MINERA B}

Coordenadas

\begin{tabular}{cccc}
\hline WGS 84 & E & N & h(m.s.n.m.) \\
\hline & 745300 & 9549362 & 1894 \\
\hline
\end{tabular}

Número total de personas que trabajan en la mina: 11 personas; 4 en cada frente; 1 persona adicional que ayuda en los 2 frentes, en 2 turnos de trabajo.

\section{LABOR MINERA C}

Coordenadas

\begin{tabular}{cccc}
\hline WGS 84 & $\mathrm{E}$ & $\mathrm{N}$ & $\mathrm{h}$ (m.s.n.m.) \\
\hline & 745251 & 9549583 & 1820 \\
\hline
\end{tabular}

Número total de personas que trabajan en la mina: 12 personas; 4 en cada frente, en 1 turno de trabajo. Para el análisis de costos comparativos para las tres 
labores mineras se toma en cuenta un frente de trabajo y un turno (ver Tablas 1-7).

\section{DISCUSIóN}

El estudio de análisis de costos muestra a tres labores mineras del sector de Nambija con diferencias muy marcadas desde el punto de vista del desarrollo técnico y económico. Las tres labores pertenecen a un mismo sector minero, sin embargo, poseen diferentes grados de tecnificación.

La mina A corresponde a una labor con un solo frente de trabajo, el personal de trabajo son los mismos socios o dueños, los equipos son limitados para desarrollar todos los trabajos, dependen del alquiler de un compresor; estos aspectos hacen que el trabajo de explotación se realice por tarea y no es posible tareas simultaneas, por lo cual el mismo grupo que realiza la perforación y voladura, es el mismo que realiza el acarreo y transporte y demás tareas mencionadas en el análisis.

La mina B es una labor minera que cuenta con personal asignado a tareas específicas, es decir cuenta con trabajadores con experiencia para cada tarea, cuenta con compresor propio que funciona a diésel, utiliza winche de izaje de material que forma un cuello de botella en el desalojo, la ventilación es natural debido a las conexiones de sus galerías.

La mina $\mathrm{C}$ cuenta con un compresor eléctrico que demanda una fuerte inversión inicial que se compensa con el costo horario en perforación, voladura y ventilación, adicionalmente cuenta con carros adaptados con capacidad de 3 toneladas en el transporte de material.

El estudio de análisis de costos en las tres labores mineras fue posible al considerar un solo frente de trabajo en cada mina.

A continuación, se muestra una comparación de los costos por cada labor minera y por tarea (ver Gráfico 1 y Tabla 8 ).

La tarea de limpieza, cargado y acarreo representa el mayor costo dentro del ciclo minero, se relaciona directamente al método de explotación utilizado; si es ascendente o descendente a favor de la gravedad, la distancia a la cual se encuentra el frente de trabajo, al uso de maquinaria y equipos influenciados por las condiciones de las vías y las dimensiones de las galerías de transporte.

La labor minera $\mathrm{C}$ utiliza autos adaptados como vagones, realiza mantenimiento de vías y tienen una sección mejorada para los carros de mina lo que ha permitido mejorar los tiempos en la tarea y aumentar su productividad.

La tarea de perforación y la tarea de voladura siguen dentro de los costos representativos del ciclo minero, que relacionan sus costos al uso de la maquinaria, equipos, y explosivos.

La siguiente tarea representativa en costos es la tarea de ventilación que se caracteriza por el uso del aire de los compresores para despejar los gases producto de la voladura.

La labor B no usa el compresor, dejan que el aire se ventile naturalmente debido a su gran cantidad de conexiones de galerías que presenta su labor minera.

Las tareas auxiliares son las que ocupan el siguiente lugar en los costos.

Cabe señalar que el costo en la tarea de sostenimiento o entibamiento no se contempló en ninguna de las tres labores mineras, debido a que, en el frente de trabajo analizado, la dureza de la roca no necesitaba de sostenimiento, lo que se evidenció durante el levantamiento de información.

\section{CONCLUSIONES}

El presente estudio muestra los costos horarios y totales calculados en tres labores mineras con diferente grado de tecnificación en el sector de Nambija.

Se evidencia que la tarea del ciclo minero de trabajo que demanda mayor utilización de personal, maquinaria, insumos, tiempo y costos, es la tarea de limpieza, cargado y acarreo que corresponde en promedio al $40,87 \%$ de los costos totales.

Seguido de la tarea de perforación con un promedio de $22,25 \%$ de los costos totales. Mientras que la tarea de voladura es un promedio de $21,12 \%$ de los costos totales.

Al tener en cuenta los factores técnicos y operativos para el cálculo de costos en cada tarea del ciclo minero de trabajo, este estudio provee información necesaria para que los mineros, socios o dueños conozcan los valores económicos que invierten y qué actividad minera demanda mayor costo.

Esto permite determinar puntos críticos para optimización no solo en el aspecto económico, sino también en el técnico.

Este estudio brinda información que ayudará al minero a conocer los factores económicos considerados en el desarrollo de su proyecto, conocer el tiempo, costo horario y costo total que demanda la actividad minera, así como contar con una herramienta para la toma de decisiones.

\section{RECOMENDACIONES}

Es importante que en las minas estudiadas se preste especial atención a la tarea de limpieza, cargado y acarreo de material, para buscar la optimización del tiempo, aplicar un adecuado diseño en las dimensiones de sus galerías, mantener el radio de giro en las curvas de las galerías acordes a las dimensiones de los equipos de 
Tabla 1. Parámetros técnicos y cálculo de costos en perforación

\begin{tabular}{|c|c|c|c|}
\hline Parámetros en perforación & Mina A & Mina B & Mina C \\
\hline Número de frentes de trabajo (unidades) & 1 & 2 & 2 \\
\hline \multirow[t]{2}{*}{ Compresor (unidades) } & 1 & 1 & 1 \\
\hline & $\begin{array}{c}\text { Alquilado a } \\
\text { diésel }\end{array}$ & Propio a diésel & $\begin{array}{l}\text { Propio } \\
\text { eléctrico }\end{array}$ \\
\hline \multirow[t]{2}{*}{ Martillo perforador (unidades) } & 1 & 1 & 2 \\
\hline & Modelo: YT27 & Modelo: YT27 & Modelo: YT27 \\
\hline Número de Barrenos integrales & 2 & 4 & 6 \\
\hline Profundidad de perforación (m) & 1,2 & 1,8 & 1,5 \\
\hline Diámetro de perforación (pulgadas) & 1 & $1 \frac{1 / 2}{2}$ & $1 \frac{1 / 2}{2}$ \\
\hline Número de barrenos por sección (unidades) & 15 & 20 & 20 \\
\hline Sección de perforación $\left(\mathrm{m}^{2}\right)$ & 1,8 & 2,7 & 2,7 \\
\hline Mano de obra utilizada & 2 & 2 & 4 \\
\hline Tiempo promedio de perforación por cada barreno (min) & 4 & 5 & 8 \\
\hline Tiempo promedio total ocupado en la tarea de perforación (h) & 1 & 1,7 & 2,7 \\
\hline Índice de utilización de personal & 1 & 1 & 1 \\
\hline Cálculo de costos en perforación & Mina A & Mina B & Mina C \\
\hline Costo total por personal (USD/h) & 7,78 & 8,89 & 11,11 \\
\hline Costo total por equipo de perforación (USD/h) & 26,21 & 14,19 & 21,64 \\
\hline Costo total por la tarea de perforación (USD) & 33,99 & 38,47 & 87,34 \\
\hline
\end{tabular}

Tabla 2. Parámetros técnicos y cálculo de costos en voladura

\begin{tabular}{|c|c|c|c|}
\hline Parámetros en voladura & Mina A & Mina B & Mina C \\
\hline Número de frentes de trabajo (unidades) & 1 & 2 & 2 \\
\hline Mano de obra utilizada & 2 & 2 & 4 \\
\hline Longitud del barreno cargado (m) & 1,1 & 1,7 & 1,5 \\
\hline Número de barrenos cargados (unidades) & 14 & 20 & 20 \\
\hline Número de barrenos descargados (unidades) & 1 & 1 & 0 \\
\hline Carga de fondo (unidades) & 1 & 1 & 1 \\
\hline Fulminante (N. $\left.{ }^{\circ} 08\right)$ & 1 & 1 & 1 \\
\hline Tipo de explosivo & $\begin{array}{c}\text { Explogel III } \\
\left(7^{\star} 11 / 8\right)\end{array}$ & $\begin{array}{c}\text { Explogel III } \\
\left(7^{\star} 1^{1 / 8}\right)\end{array}$ & $\begin{array}{l}\text { Explogel III } \\
\left(7^{\star} 1^{1 / 8}\right)\end{array}$ \\
\hline Carga de columna (Kg) & 0,3 & 0,4 & 0,6 \\
\hline Tipo & $\begin{array}{l}\text { Nitrato de } \\
\text { amonio }\end{array}$ & $\begin{array}{l}\text { Nitrato de } \\
\text { amonio }\end{array}$ & $\begin{array}{l}\text { Nitrato de } \\
\text { amonio }\end{array}$ \\
\hline Mecha lenta (m) & 1,4 & 1,9 & 1,6 \\
\hline Retacado (m) & 0,6 & 0,8 & 0,6 \\
\hline
\end{tabular}


Tiempo promedio del ciclo de cargado de un barreno (min)

2

0,5

0,75
3

1

1
2

0,75

\begin{tabular}{lccc}
\hline Cálculo de costos en voladura & Mina A & Mina B & Mina C \\
\hline Costo total por personal (USD/h) & 7,78 & 8,89 & 11,11 \\
Costo total por insumos para la voladura (USD/h) & 65,29 & 49,62 & 74,88 \\
Costo total por la tarea de voladura (USD) & 34,1 & 58,51 & 57,33 \\
\hline
\end{tabular}

Tabla 3. Parámetros técnicos y cálculo de costos en ventilación

\begin{tabular}{lccc}
\hline Parámetros en ventilación & Mina A & Mina B & Mina C \\
\hline Mano de obra utilizada & 1 & 0 & 1 \\
Longitud de la galería hasta el frente de trabajo (m) & 90 & 300 & 500 \\
Número de niveles de trabajo & 1 & 3 & 2 \\
$\begin{array}{l}\text { Tiempo promedio del ciclo de ventilación artificial en el frente de } \\
\text { trabajo (h) }\end{array}$ & 1 & 0 & 1 \\
Tiempo total efectivo utilizando el compresor (h) & 0,5 & ventilación & 0,5 \\
& 0,25 & 0,25 & 0,25 \\
Índice de utilización de personal & & & \\
\hline & Mina A & Mina B & Mina C \\
\hline Cálculo de costos en ventilación & 3,33 & 0 & 3,33 \\
\hline Costo total por personal (USD/h) & 20 & 0 & 10,58 \\
Costo total por insumos para la ventilación (USD/h) & 13,33 & 0 & 8,63 \\
Costo total en la tarea de ventilación (USD) & &
\end{tabular}

Tabla 4. Parámetros técnicos y cálculo de costos en saneamiento

\begin{tabular}{|c|c|c|c|}
\hline Parámetros en saneamiento del frente de trabajo & Mina A & Mina B & Mina C \\
\hline Mano de obra utilizada & 1 & 1 & 1 \\
\hline Herramienta utilizada (unidades) & 1 & 1 & 1 \\
\hline Tipo de herramienta & $\begin{array}{c}\text { Barreta } \\
\text { alargada con } \\
\text { punta refor- } \\
\text { zada }\end{array}$ & $\begin{array}{l}\text { Barreta alarga- } \\
\text { da con punta } \\
\text { reforzada }\end{array}$ & $\begin{array}{c}\text { Barreta } \\
\text { alargada } \\
\text { con punta } \\
\text { reforzada }\end{array}$ \\
\hline Tiempo promedio en saneamiento del frente de trabajo $(\mathrm{h})$ & 0,5 & 0,5 & 0,5 \\
\hline Índice de utilización de personal & 0,25 & 0,33 & 0,25 \\
\hline Cálculo de costos en saneamiento del frente de trabajo & Mina A & Mina B & Mina C \\
\hline Costo total por personal (USD/h) & 3,33 & 3,89 & 3,89 \\
\hline Costo total por insumos 15\% de la mano de obra (USD/h) & 0,5 & 0,58 & 0,58 \\
\hline Costo total en la tarea de saneamiento (USD) & 1,92 & 2,24 & 2,24 \\
\hline
\end{tabular}


Tabla 5. Parámetros técnicos y cálculo de costos en limpieza, cargado y acarreo

\begin{tabular}{lccc}
\hline Parámetros en limpieza, cargado y acarreo & Mina A & Mina B & Mina C \\
\hline Mano de obra utilizada & 4 & 6 & 3 \\
Número de vagones (unidades) & 1 & 2 & 5 \\
Cantidad de viajes en vagones desalojados (unidades) & 7 & 15 & 3 \\
Capacidad de los vagones (t) & 1 & 1 & 1,7 \\
Tiempo promedio del ciclo de acarreo del mineral a la boca mina (h) & 0,47 & 5,33 & 5 \\
Tiempo promedio en limpieza, cargado y acarreo (h) & 3,3 & 1 & 1 \\
Índice de utilización de personal & 1 & 1 \\
\hline
\end{tabular}

\begin{tabular}{lccc}
\hline Cálculo de costos en limpieza, cargado y acarreo & Mina A & Mina B & Mina C \\
\hline Costo total por personal (USD/h) & 13,33 & 23,33 & 11,67 \\
Costo total por uso de herramientas (palas, barras) (USD/h) & 0,52 & 0,41 & 0,53 \\
Costo total por uso de transporte (vagón/ carro) (USD/h) & 0,71 & 0,64 & 7,54 \\
Costo total del sistema de izaje con winche (USD/h) & 0 & 4,25 & 0 \\
Costo total en la tarea de limpieza, cargado y acarreo (USD) & 48,06 & 157,46 & 98,65 \\
\hline
\end{tabular}

Tabla 6. Parámetros técnicos y cálculo de costos en tareas auxiliares

\begin{tabular}{lccc}
\hline Parámetros en tareas auxiliares & Mina A & Mina B & Mina C \\
\hline Mano de obra utilizada & 2 & 2 & 30 \\
Tiempo rpoemdio en colocación de tubería & 30 & 30 & $2,1,1,2$ \\
Herramienta utilizada & $2,1,1,2$ & $2,1,1,2$ & Palas, \\
Tipo de herramienta & Palas, combo, & Palas, \\
& barra, cuñas combo, combo, & barra, \\
& & cuñas cuñas & cuñas \\
Tiempo total promedio en instalación $(h)$ & 0,75 & 0,75 & 0,75 \\
Indice de utilización de personal & 0,5 & 0,5 & 0,5 \\
\hline
\end{tabular}

\begin{tabular}{lccc}
\hline Cálculo de costos en tareas auxiliares & Mina A & Mina B & Mina C \\
\hline Costo total por personal (USD/h) & 6,67 & 6,67 & 6,67 \\
Costo total por uso de herramientas (palas, barras) (USD/h) & 0,52 & 0,41 & 0,53 \\
Costo total por insumos requeridos en las actividades auxiliares (USD/h) & 9,13 & 9,67 & 9,6 \\
Costo total en la tarea auxiliar (USD) & 12,24 & 12,56 & 12,59 \\
\hline
\end{tabular}

Tabla 7. Cálculo de costos adicionales

\begin{tabular}{lccc}
\hline Cálculo de costos adicionales & Mina A & Mina B & Mina C \\
\hline Costo por uso de energía eléctrica (USD/h) & 0,67 & 1,33 & 1,92 \\
Costo por equipo de protección (USD/h) & 0,9 & 2,5 & 2,5 \\
\hline
\end{tabular}


Gráfico 1. Costos totales por tarea de cada labor minera estudiada

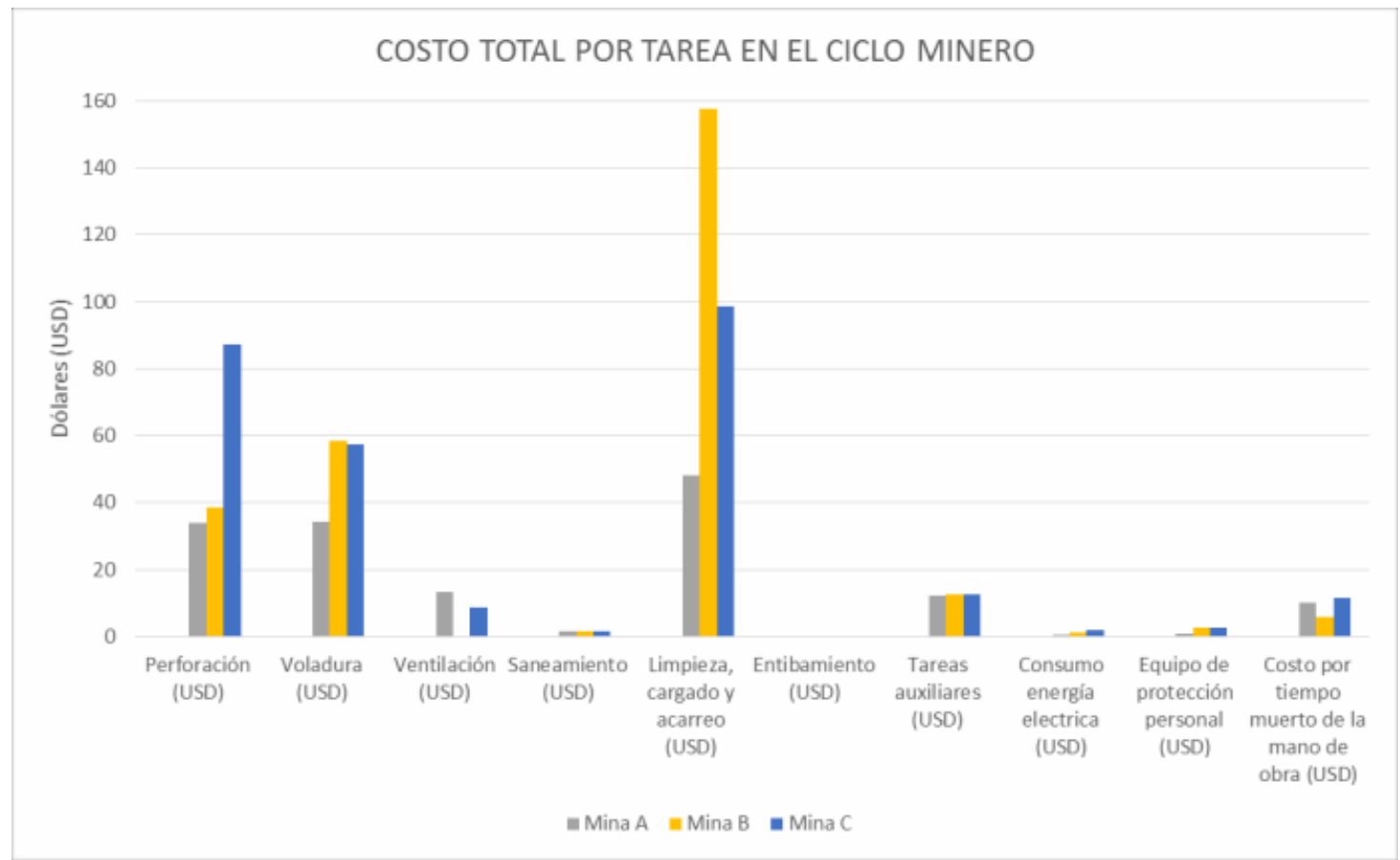

Tabla 8. Costos totales en el ciclo de trabajo en las tres labores mineras

\begin{tabular}{llll}
\hline Tarea & Mina A & Mina B & Mina C \\
\hline Perforación (USD) & 33,99 & 38,47 & 87,34 \\
Voladura (USD) & 34,1 & 58,51 & 57,33 \\
Ventilación (USD) & 13,33 & 0,00 & 8,63 \\
Saneamiento (USD) & 1,44 & 1,44 & 1,44 \\
Limpieza, cargado y acarreo (USD) & 48,06 & 157,46 & 98,65 \\
Entibamiento (USD) & 0,00 & 0,00 & 0,00 \\
Tareas auxiliares (USD) & 12,24 & 12,56 & 12,59 \\
Consumo energía eléctrica (USD) & 0,67 & 1,33 & 1,92 \\
Equipo de protección personal (USD) & 0,90 & 2,47 & 2,47 \\
Costo por tiempo muerto de la mano de obra (USD) & 10,28 & 5,94 & 11,53 \\
Costo total & $\mathbf{1 5 5 , 0 1}$ & $\mathbf{2 7 8 , 1 9}$ & $\mathbf{2 8 1 , 9}$ \\
\hline
\end{tabular}

transporte utilizados, adecuado diseño de buzones de trasiego de material, limpieza que permita liberar los obstáculos durante el transporte de vagones sin generar retrasos o interrupciones y el correcto mantenimiento y nivelación de vías.

Con respecto a la perforación y voladura, se debe tener un diseño adecuado de la malla de perforación, adecuada distribución de explosivos para obtener material condicionado, con el fin de facilitar el desa- rrollo y mejora de la actividad de limpieza, cargado y acarreo de material arrancado.

El costo en la tarea de ventilación en el sector de Nambija se puede manejar en su mayoría apoyados de la ventilación natural debido a las conexiones existentes entre galerías.

De ser el caso en condiciones donde la roca presente características o durezas débiles, por la presencia de fallas o fracturas entre otras, se debe considerar también los costos de la tarea de entibamiento o sostenimiento. 


\section{NOTAS}

1. Instituto Nacional de Investigación Geológico Minero Metalúrgico (INIgEMm, 2012). El Distrito Minero Nambija comprende las minas de Cumay, La Fortuna, Cambana, Campanillas, Nambija, Guaysimi, Tumi, Sultana y otras menores ocurrencias. La geología de estos depósitos y el tipo de mineralización es similar con ocurrencias explotables auríferas relacionadas con skarn e indicios de mineralización $\mathrm{Cu}-\mathrm{Au}$ y $\mathrm{Cu}-\mathrm{Mo}$ relacionadas con pórfidos.

2. Instituto Nacional de Investigación Geológico Minero Metalúrgico (INIGEMM, 2012). La principal mineralización en el Distrito Minero Nambija es de oro, pero se reportan asociaciones porfíricas de $\mathrm{Cu}-\mathrm{Au}$ y $\mathrm{Cu}-\mathrm{Mo}$. El oro nativo aparece generalmente libre y tienen una pureza del $90 \%$, con un porcentaje de plata de 7-10\%. El oro puede presentarse en granos de varios milímetros hasta de tamaño microscópico.

3. INTERCADE Consultancy \& Training, (Reátegui Ordoñez, 2018). El proceso minero se inicia con la exploración de áreas donde, por las condiciones geológicas, se estima que existan depósitos de minerales de interés económico. En esta fase básicamente se estima los recursos minerales existentes.

4. Instituto Tecnológico GeoMinero de España, (Arteaga Rodríguez, et al., 1991). Cualquier proyecto minero, independientemente de la fase en la que se encuentre - investigación, evaluación o desarrollo- presenta una dimensión económica ineludible que debe analizarse antes de asignar los recursos necesarios para llevarlo a cabo.

5. INTERCADE Consultancy \& Training, (Reátegui Ordoñez, 2018). Si los recursos minerales, luego de un análisis técnico-económico, tienen un valor económico real, se convierte en reservas y se inicia la fase de proyecto, en donde se determina todas las variables necesarias para extraer y beneficiar las reservas minerales.

6. Instituto Tecnológico GeoMinero de España, (Arteaga Rodríguez, et al., 1991). Los costos de capital fijo se refieren a los fondos necesarios para la adquisición de terrenos, maquinaria, edificaciones e instalaciones, etc.

7. Instituto Tecnológico GeoMinero de España, (Arteaga Rodríguez, et al., 1991). El capital circulante representa el dinero necesario para comenzar la operación y asumir las obligaciones subsiguientes durante la puesta en marcha del proyecto.

8. INTERCADE Consultancy \& Training, (Reátegui Ordoñez, 2018). Finalmente se inicia la fase operativa donde, de acuerdo a un planeamiento adecuado, permite extraer y beneficiar de la forma más eficiente las reservas.

9. Instituto Tecnológico GeoMinero de España, (Arteaga Rodríguez, et al., 1991). El resultado de la explotación se obtiene por diferencia entre los ingresos por la venta de minerales y sus costes de producción.
10. Alianza Mundial de Derecho Ambiental (Elaw, 2010). La molienda es una de las actividades más costosas del beneficio de minerales y resulta en partículas muy finas que pueden permitir una mejor extracción del metal.

11. Banco Central del Ecuador, 2018. Desde junio 2016 el BCE inició un programa de compra del oro proveniente de la pequeña minería y minería artesanal, de forma directa o por intermedio de agentes económicos públicos o privados autorizados por la entidad. La compra se hace a precio internacional, IVA $0 \%$ y se transfiere máximo en 48 horas. Adicionalmente esta entidad cuenta con una oficina técnica encargada de ese proceso en la ciudad de Machala.

12. Universidad Nacional de Colombia; sede Medellín, Boletín de Ciencias de la Tierra, (Jiménez, et al., 2006). Los factores críticos lo componen las etapas mineras de desarrollo, preparación y explotación, las cuales se subdividen en operaciones mineras (perforación, voladura, Sostenimiento, Cargue y Transporte), siendo cerca del $70 \%$ de los costos operativos de extraer una tonelada de mineral.

13. Universidad Nacional de Piura, (Vega Farfán, 2013). Los costos de propiedad existen aun cuando la máquina no se encuentra trabajando (capital Inmovilizado).

14. Universidad Nacional de Piura, (Vega Farfán, 2013). El costo horario de operación minera considera los siguientes aspectos: consumos: combustibles y energía, lubricantes (aceites y grasas), filtros (de aire, gasolina y aceite), materiales de desgaste, reparaciones, neumáticos o tren de rodaje, operadores.

15. Universidad Nacional de Colombia; sede Medellín, Boletín de Ciencias de la Tierra, (Jiménez, et al., 2006). El costo real se halla con base en la información tomada en campo, como, por ejemplo, número de trabajadores por turno, número de turnos, producción por turnos, relación entre volumen de estéril y mineral removido, cantidad de insumos requeridos, inversión en infraestructura, costo/tonelada, entre otros.

\section{REFERENCIAS}

Agencia de Regulación y Control Minero (ARCOM), 2013. Resolución N. ${ }^{\circ}$ 001-ins-dir- arcom-2013, Quito: s. n.

Alianza Mundial de Derecho Ambiental (ELAW), 2010. Guía para evaluar EIAs de proyectos mineros, s. l.: s. n. Arteaga Rodríguez, R. y otros, 1991. Manual de evaluación técnico económica de proyectos mineros de inversión, Madrid: s. n.

Banco Central del Ecuador, 2018. BCE inaugura oficina en Machala para comercializar oro con mineros pequeños y artesanales. https:// www.bce.fin.ec/index.php/boletines-de- 
prensa-archivo/item/1039-bce-inaugura-oficinaen-machala-para-comercializar-oro-con-minerospequenos-y-artesanales

Instituto Nacional de Investigación Geológico Minero Metalúrgico inigemm, 2012. Geología Regional y Local de Nambija. Proyecto Rehabilitación Nambija, Quito: s.n.

Jiménez, P., Indhira, M., Molina, E. y Jorge, M., 2006. Pro- puesta de medición de la productividad en minería de oro vetiforme y reconocimiento de estándares productivos sostenibles. Boletín Ciencias de la Tierra; núm. 19 2006, pp. 73-86 0120-3630.

Ley de Minería, Ecuador, 2018. Ley de Minería, Quito: s. n. Ministerio de Minería, 2015. Resolución N. ${ }^{\circ}$ mm-czm-S2015-0878-rm. Zamora (Zamora Chinchipe): s. n.

Reátegui Ordoñez, C., 2018. Análisis global de costos en operaciones de minería subterránea, s. l.: s. n.

Vega Farfán, J. L., 2013. Curso: Control de operaciones mineras, Piura: s. n. 